\title{
Motivation and motivational climate among elite hammer throwers
}

\author{
Ottó Benczenleitner, József Bognár, László Révész, Judit Paksi, István Csáki, Gábor Géczi \\ Semmelweis University Faculty of Physical Education and Sport Sciences Budapest, Hungary
}

\section{Summary}

Study aim: The purpose of the study was to explore the characteristics of the motivational factors and the motivational climate of elite hammer throwers in the most prestigious and most successful club in Hungary.

Material and methods: The Sport Motivational Scale (SMS) and the Perceived Motivational Climate in Sport Questionnaire (PMCSQ) were used to assess the motivation and motivational climate among hammer throw athletes $(\mathrm{N}=23)$. Data were analysed with descriptive statistical methods. The independent sample t-test was applied for the determination of differences as related to success level, age, and gender. Also, correlation was used to find out what relationships exist among the variables.

Results: Amotivation tends to be at low levels $(\mathrm{M}=2.48 \pm 1.40)$, while extrinsic motivation $(\mathrm{M}=4.68 \pm 0.79)$ and intrinsic motivation $(M=4.40 \pm 0.95)$ can be considered to be at medium levels in the sample. There were no differences in motivation between elite and non-elite athletes and between athletes under and over 18 years of age. However, male throwers had a significantly higher level of intrinsic motivation $(\mathrm{t}=2.941, \mathrm{p}=.008)$ and a significantly lower level of amotivation $(t=-2.428, p=0.025)$ than female athletes. The sample had higher values of Task orientation $(3.97 \pm 0.40)$ than Ego orientation (2.63 \pm 0.47$)$. There were no differences detected in success level, gender, and age in the athletes' motivational climate. The correlation in all cases seems to be generally low.

Conclusions: The coach of this successful club focuses on creating a positive climate in which the athletes' skill levels, goal orientation, age, and gender are matched with challenges and expectations. The relatively few differences in motivation and motivational climate prove that the coach provides continuous support on both the individual and group level. The coach does it not only with the athletes' skills and conditioning, but also with their motivation and motivational climate.

Key words: motivation, elite athletes, hammer throwers

\section{Introduction}

The selection process of athletes and their talent management have considerable importance in achieving athletic excellence. Not only are exceptional motor abilities and skills searched for, but driving motives, motivation, and goal orientation also receive increasing attention from sport researchers, coaches, and managers [13]. It is evident today that the presence of certain physical and anthropometric abilities and skills does not seem sufficient enough to make purposeful decisions on the selection process and development programs.

It is well documented that certain psychological factors are required for high achievement in sport [12]. Persistence, effort, and motivation seem to be significant issues in this process, so it is worthwhile to further explore in different areas and fields of sport [3]. One important question in elite sport is to find out how motives are characterised in the process of driving sport behaviours and how these motives can be modified by coaches [4]. It is well known that motivation is responsible for initiating and maintaining effort and also for a high level of athletic behaviour and performance. Consequently, motivational characteristics and profiles have significant implications for athletic programs, talent identification and development, and for coaches' day-to-day work with athletes.

Motivation, dedication, effort, and commitment are those critical components of an athlete that are necessary for mastering skills and building success. Intrinsic motivation indicates a volunteer behaviour for engaging in an activity purely for satisfaction; on the other hand, extrinsic motivation affects athletic behaviour in a way that does not solely serve the satisfaction of needs but rather the adjustment to the expectations of the environment. Athletes with high intrinsic motivation tend to have a higher level of self-appreciation and are generally more successful in sport than their less motivated peers [28].

Both the climate and the expectations of coaches can considerably impact and eventually determine athletic success. The coach-athlete relationship, as well as the climate provided by and maintained by the coach, have significant influences on the motivational interest of an athlete [16, 28]. 
Two types of coach-initiated motivational climates have been identified in the literature [1]. Athletes with Task orientation use personal, long-term, and flexible standards for achieving success and they mostly focus on what they can do to improve their skills [9]. The Taskinvolving climate principally measures how athletes perceive coaches' emphasis on their effort and improvement [14]. On the other hand, athletes with Ego orientation focus on demonstrating more competence and also they make judgments about subjective success through the social comparison processes [19]. An Ego-involving climate tends to measures athlete's perceptions of punishment for mistakes, recognition of only the best players, and Intrateam competition among athletes.

Research studies focusing on hammer throwers have a tendency to focus mostly on velocity, turns, distance, and other technical factors connected to the sport [5, 7, 23]. To become exceptional in this event has been connected mostly with strength training [17], repetition, high levels of technical skills [10], and specific training programs [18]. There are very few studies focusing on elite throwers' psychological characteristics, including motivation and climate. Also, it seems relatively rare that every athlete from a very successful club has taken part in a motivational study. Hence, it seems important to examine the motivational characteristics of elite throwers and the link between motivational climate and motivation in athletics especially as they relate to this event.

In this study, our aim was to examine the characteristics of motivational factors and the motivational climate of elite hammer throwers in a successful club (Dobó SE) in Hungary. Since this club only focuses on throwing events in athletics, the context is unique and makes it worthwhile to explore using this approach. This club involves numerous Olympic, World, and European Championship winners and medallists, which makes this club exceptionally successful in throwing events. It is our expectation that the level of motivation is generally high and the motivational climate is more Task-oriented than Ego-oriented. The differences in motivation and climate as related to success level, gender, and age is expected to be minimal. We also anticipate a relationship between motivation and motivational climate.

\section{Material and Methods}

Two surveys were conducted among elite hammer thrower athletes to assess their motivation and motivational climate. Altogether there were 23 active hammer throwers in this highly prestigious club in Szombathely, Hungary. All of them were included in the study $\left(M_{\mathrm{age}}=20.43 \pm 6.62\right)$. The same well-qualified and experienced coach was responsible for the whole group regardless of their success level, age, and gender. These athletes had a relatively long-lasting participation in this sport $(\mathrm{M}=8.86 \pm 6.37)$. After receiving ethical approval for this study, the club was contacted with a request for the involvement of every athlete in the study. Parental consent was obtained for athletes under 18 years of age.

The throwers were considered elite athletes because they all demonstrated performance excellence in both training and competition. The sample was divided into groups in relation to their achievement and performance levels, age, and gender. Athletes considered highly successful (elite) were those who earned points (1st-8th places) in the Olympic Games, World Championships, or European Championships ( $\mathrm{n}=11)$. The less successful category consisted of 12 athletes. Considering age: there were 13 athletes under the age of 18; 10 were older than 18 years old. Gender distribution was 12 male and 11 female athletes in the study.

Among the successful athletes, there were several Olympic, World, and European champions and medallists. In the last two decades, athletes representing this club have won 2 Olympic gold medals, 5 World Championship gold medals, and 5 European Championship gold medals. Due to this club's emphasis on talent identification and management, throwers from this club have in the last decade won 4 gold medals in the Junior World Championships, 4 gold medals the Junior European Championships, 6 gold medals in the Youth World Championships, and 1 gold medal in the Youth European Championship.

The two questionnaires were distributed by the coach in the middle of the competitive season in 2012. Every athlete completed the questionnaires at the beginning of a session. Motivation of athletes was measured with the Sport Motivational Scale (SMS) [20], which contained 28 items and three sub-scales with the purpose of determining components of intrinsic motivation, extrinsic motivation, and amotivation. A 7-point Likert scale was employed in this survey. The Hungarian version of Sport Motivation Survey was developed by Tsang and colleagues [26] and had an acceptable level of internal reliability and also internal consistency (0.65-0.85).

For assessing the motivational climate of elite throwers, the Perceived Motivational Climate in Sport Questionnaire (PMCSQ) was employed [19]. This survey measures perceptions of motivational climate and is characterised by two higher order dimensions of the achievement goal frameworks: Task-involving and Ego-involving climate. Cooperative learning, important role, and effort/ improvement subscales measure Task-involving climate, whereas punishment for mistakes, unequal recognition, and Intrateam member rivalry subscales measure Ego-involving climate. The 33item questionnaire consists of closed-ended questions, and each statement had to be rated on a 1-to-5-point scale. 
The Hungarian version of the HPMCSQ-2 was validated by Révész and colleagues [22] with adequate reliability, and its internal consistency ranged from 0.65-0.92. Data were analysed with descriptive statistical methods. The independent sample t-test was applied for the determination of differences between groups. Also, correlation was used to find out what relationship exists among the variables. SPSS 19.0 for Windows was utilised for all statistical calculations.

\section{Results}

When analysing sport motivation scale, it can be seen that amotivation tends to be at a low level $(M=2.48 \pm 1.40)$. Extrinsic motivation ( $\mathrm{M}=4.68 \pm 0.79)$ and intrinsic motivation $(\mathrm{M}=4.40 \pm 0.95)$ can be considered at a medium level in the 1-7 scale (Table 1). According to the independent sample $\mathrm{t}$-test, there were no differences in any of the 3 subscales of motivation between highly successful and less successful athletes. No differences were found between athletes under and over 18 years of age. Conversely, male throwers had a significantly higher level of intrinsic motivation $(t=2.941$, $\mathrm{p}=0.008)$ and a significantly lower level of amotivation $(\mathrm{t}=-2.428 ; \mathrm{p}=0.025)$ than female athletes.

When focusing on perceived motivational climate, Ego orientation (2.63 \pm 0.47$)$ seems to be considerably lower than their Task orientation (3.97 \pm 0.40$)$ (Table 1). From the subscales of Task orientation, Important role has the highest value (4.55 \pm 0.85$)$ and Cooperative learning the lowest value (3.86 \pm 0.57$)$. The values of Effort/improvement (3.86 \pm 0.57$)$ is in the middle of the 1-5 scale. In the Egoorientation subscale, both Punishment for mistakes $(2.35 \pm 0.46)$ and Unequal recognition $(2.54 \pm 0.62)$ have relatively low values and Intrateam member rivalry value is bit higher than a medium level range (3.37 \pm 0.66$)$. When comparing and contrasting the subscales of PMCSQ-2 as related to different variables, there were no differences detected in success level, gender, and age.

Table 2 shows the correlation coefficients among all the subscales. The correlation in all cases seems to be generally low. Correlation coefficients were at a significant level in a few instances (Table 2); however, they all seem to be at a low level of correlation (0.457-0.535) and so should not be considered meaningful.

Table 1. Descriptive statistics (Mean \pm Standard Deviation) considering success, gender and age as an independent variables $(\mathrm{N}=23)$

\begin{tabular}{lccccccc} 
& Total & $\begin{array}{c}\text { Nonsuccess } \\
(\mathrm{n}=11)\end{array}$ & $\begin{array}{c}\text { Success } \\
(\mathrm{n}=12)\end{array}$ & $\begin{array}{c}\text { Male } \\
(\mathrm{n}=12)\end{array}$ & $\begin{array}{c}\text { Female } \\
(\mathrm{n}=11)\end{array}$ & $\begin{array}{c}\text { Under 18 } \\
\text { years of age } \\
(\mathrm{n}=13)\end{array}$ & $\begin{array}{c}\text { Over } 18 \text { years } \\
\text { of } \\
\text { age }\end{array}$ \\
\hline Amotivation & $2.48 \pm 1.40$ & $2.25 \pm 1.42$ & $2.67 \pm 1.40$ & $1.87 \pm 1.19^{*}$ & $3.15 \pm 1.33^{*}$ & $2.48 \pm 1.28$ & $2.50 \pm 1.61$ \\
Intrinsic motivation & $4.40 \pm 0.95$ & $4.79 \pm 0.94$ & $4.60 \pm 0.68$ & $5.09 \pm 0.87 *$ & $4.25 \pm 0.37 *$ & $4.77 \pm 0.98$ & $4.57 \pm 0.47$ \\
Extrinsic motivation & $4.68 \pm 0.79$ & $4.26 \pm 1.07$ & $4.51 \pm 0.87$ & $4.51 \pm 0.92$ & $4.28 \pm 1.00$ & $4.51 \pm 1.06$ & $4.26 \pm 0.80$ \\
\hline Task & $3.97 \pm 0.40$ & $4.13 \pm 0.35$ & $3.85 \pm 0.41$ & $4.06 \pm 0.45$ & $3.87 \pm 0.34$ & $4.11 \pm 0.36$ & $3.79 \pm 0.40$ \\
Cooperative & $3.86 \pm 0.57$ & $4.00 \pm 0.51$ & $3.76 \pm 0.61$ & $3.85 \pm 0.51$ & $3.87 \pm 0.66$ & $4.04 \pm 0.60$ & $3.65 \pm 0.48$ \\
learning & $4.55 \pm 0.85$ & $4.88 \pm 0.86$ & $4.31 \pm 0.79$ & $4.72 \pm 0.99$ & $4.37 \pm 0.67$ & $4.70 \pm 0.89$ & $4.36 \pm 0.82$ \\
Important role & $4.26 \pm 1.36$ & $4.34 \pm 0.36$ & $4.20 \pm 0.36$ & $4.38 \pm 0.41$ & $4.11 \pm 0.23$ & $4.36 \pm 0.30$ & $4.13 \pm 0.40$ \\
\hline Effort/Improvement & $2.63 \pm 0.47$ & $2.52 \pm 0.37$ & $2.72 \pm 0.54$ & $2.61 \pm 0.40$ & $2.66 \pm 0.57$ & $2.58 \pm 0.56$ & $2.70 \pm 0.39$ \\
Ego & $2.35 \pm 0.46$ & $2.16 \pm 0.42$ & $2.50 \pm 0.45$ & $2.31 \pm 0.43$ & $2.38 \pm 0.51$ & $2.27 \pm 0.54$ & $2.44 \pm 0.34$ \\
Punishment for & $2.54 \pm 0.62$ & $2.42 \pm 0.30$ & $2.61 \pm 0.76$ & $2.41 \pm 0.50$ & $2.68 \pm 0.73$ & $2.58 \pm 0.66$ & $2.50 \pm 0.61$ \\
mistakes & $3.37 \pm 0.66$ & $3.33 \pm 0.57$ & $3.41 \pm 0.73$ & $3.30 \pm 0.64$ & $3.46 \pm 0.70$ & $3.36 \pm 0.67$ & $3.40 \pm 0.68$ \\
$\begin{array}{l}\text { Unequal recognition } \\
\text { Intrateam member } \\
\text { rivalry }\end{array}$ & & & & &
\end{tabular}

Legend: * $\mathrm{p}<0 ., 05$ SMS ranges between 1-7, PMCSQ-2 ranges between 1-5.

Table 2. Correlation for all the SMS and PMCSQ-2 subscales ( $\mathrm{N}=23)$

\begin{tabular}{lcccccccc}
\hline & TASK & EGO & $\begin{array}{c}\text { Task } \\
\text { Cooperative } \\
\text { learning }\end{array}$ & $\begin{array}{c}\text { Task } \\
\text { Important } \\
\text { role }\end{array}$ & $\begin{array}{c}\text { Task } \\
\text { Effort } \\
\text { Improvement }\end{array}$ & $\begin{array}{c}\text { EGO } \\
\text { Punishment } \\
\text { for } \\
\text { mistakes }\end{array}$ & $\begin{array}{c}\text { EGO } \\
\text { Unequal } \\
\text { recognition }\end{array}$ & $\begin{array}{c}\text { EGO } \\
\text { Intrateam } \\
\text { member } \\
\text { rivalry }\end{array}$ \\
\hline $\begin{array}{l}\text { SMS } \\
\begin{array}{l}\text { Amotivation } \\
\text { SMS Extrinsic }\end{array}\end{array}$ & 0.071 & 0.246 & 0.128 & 0.038 & -0.063 & 0.132 & 0.075 & 0.235 \\
$\begin{array}{l}\text { motivation } \\
\text { SMS Intrinsic } \\
\text { motivation }\end{array}$ & 0.429 & 0.394 & 0.279 & $0.475^{*}$ & 0.273 & 0.421 & 0.279 & $0.457^{*}$ \\
\hline
\end{tabular}




\section{Discussion}

The aim of our study was to investigate the characteristics the motivation and motivational climate in hammer throw athletes as well as to investigate the differences between different success levels, age groups, and genders from a prestigious club (Dobó SE) in Szombathely, Hungary. According to our results, hammer throwers demonstrate a low level of amotivation and a medium level of extrinsic and intrinsic motivation. Extrinsic motivation is slightly higher in the sample than intrinsic motivation. It is understood that competitive athletes are aiming for medals and championship titles rather than purely for enjoyment. Selfsatisfaction, fun, and pleasure have less appearance in the demanding and lengthy training process. So the coach's role seems to have critical importance in supporting and motivating athletes throughout the seasons. This role requires planning, coaching, and communicating in a careful manner so that the large number of repetition in drills and exercise does not contribute to high level of amotivation.

Intrinsically motivated athletes enjoy participation in sport more and are less likely to drop out [29]. Due to their achievements at national and international competitions, some athletes in this study should be considered as competing at the world-class level, though still having a medium level of intrinsic motivation. As a result of considerable successes, as well as the high hopes for excellence and victories, these athletes remain in competitive sport for a long time. Factors that keep the athletes in sport even in the most demanding training sessions should be associated with the coach's personality, open communication, and high hopes for the future. Contrary to the individualistic nature of throwing events, athletes who work together towards their own goals and their peers' achievements probably facilitate their perseverance and determination in the course of training and competition.

Athletes in our study had higher values of Task orientation compared to Ego orientation. As expected, athletes perceive the coach's emphasis on their effort and improvement, which leads towards Task-involving climate. Also, studies report that people with Task orientation more often experience success than those with Ego orientation [11,24]. In Task-involving motivational climate, Important role has the highest value of all subscales, which means the coach continuously emphasizes the value of effort and the need for hard work athletes making as a contribution to the club. Important role seems to be the factor that significantly affects the motivational climate when focusing on athletes working towards their goals. It is somewhat unexpected that Effort/Important is not higher, as this sport event requires lot of efforts and highly mastered skills. The mid-range value of Cooperative learning seems to go well with the individualistic nature of throwing events; nevertheless, athletes go through similar processes in terms of skill development. Since many of the training sessions and practices take place at the same place and same time, learning from one another is a useful tool when improving or refining skills. Intrateam member rivalry has somewhat higher values in Ego orientation and helps to understand how much emphasis a coach puts on encouraging rivalry among athletes. When a club has world-class athletes, it might not easy for the rest of the team to see their chances in matching themselves to those successful athletes. Also, to minimize the gap between successful and non-successful athletes, a coach has opportunities to plan for fair competitive drills that can lead to individual challenge in each practice. Punishment for mistakes and Unequal recognition has moderate value, meaning that coaches do not punish athletes due to their mistakes and he/she tends to give fair attention to each thrower unrelated to their skills, successes, gender, or age.

Athletes with intrinsic motivation tend to persist at certain activities, select challenging tasks, give effort [8], and they are driven by perceived competence, perceived autonomy and goal achievement orientation [15]. Petherick and Weigand reported that motivation is influenced by the climate [21]. However, this study found no correlation between motivation and perceived motivational climate. The date of data collection might make meaningful difference in results. Data collection took place in the middle of the competition season of an Olympic year (that Olympic Game also proved that this club was among the best, as a gold medal was awarded to them). Future studies need to focus on how motivation and motivational climate changes in different periods of the season.

Contrary to our expectations, there were no differences between the elite and the less successful groups and also between younger and older athletes in motivation and motivational climate. Research studies support that male and female athletes exhibit similar personality characteristics [2]; however, other studies proved that male athletes have higher levels of extrinsic motivation than female athletes [21]. Contrary to our recent findings, research supports that fact that women have higher levels of intrinsic motivation than men $[9,27]$. This difference can be attributed to the alterations in the sample and sport discipline.

It is worth highlighting that the results of this study prove our earlier findings in relation to gender differences [13]. Studies concluded that elite male athletes have higher levels of intrinsic motivation and a lower level of amotivation than elite female athletes. That information can support the fact that there is a need for different coaching methods directed to motivational issues for groups with different characteristics, such as male and female athletes. 
Csíkszentmihályi [6] reported that individuals tend to be intrinsically motivated when the level of task challenge is suited to one's skills. It might be useful advice for coaches to focus on creating a positive climate in which individual athletes' skill levels, goal orientation, and gender are matched with the challenges. Coaches need to plan and design practice for individually challenging practices ensuring that their athletes could be more motivated and so more successful [25].

\section{References}

1. Ames C. (1992) Classrooms: Goals, structures, and student motivation. J. Educ. Psychol., 84: 261-271.

2. Bebestos E., P.Antonious (2003) Psychological skills of Greek badminton athletes. Percept. Motor. Skill., 97:1289-1296.

3. Bergin D.A., S.F.Habusta (2004) Goal orientations of young male ice hockey players and their parents. J. Genet Psychol., 165:383-397.

4. Bollók S., J.Takács, ; Z.S.Kalmár, B.Dobay (2011) External and internal sport motivations of young adults, Biomed. Hum. Kin., 3:101-105.

5. Brice S., K.Ness, D.Rosemond, K.Lyons, M. Davis (2008) De-velopment and validation of a method to directly measure the ca-ble force during the hammer throw. Sports Biomech. 7(2):274-287.

6. Csíkszentmihályi M. (1975) Beyond boredom and anxiety. Jossey-Bass, San Francisco.

7. Dapena J., G.McDonald (1989) A three-dimensional analysis of angular momentum in the hammer throw. Med. sci sport. exer. 21(2):206-220.

8. Deci E.L., R.J. Vallerand,, L.G.Pelletier, R.M.Ryan (1991) Motivation and education: The self-determination perspective. Educ. Psychol., 26:325-346.

9. Duda J., L.Chi, M.Newton, M.Walling, D.Catley (1995) Task and ego orientation and intrinsic motivation in sport. Internat. J. Sport Psychol., 26:40-63.

10. Dunn G., K.McGill (1991) The Throws Manual. Palo Alto, California: Track and Field News Press, Palo Alto.

11. Eccles J.S., A.Wigfield (2002) Motivational beliefs, values, and goals. Annu. Rev. of Psychol., 53:109-132.

12. Géczi G., L.Révész, J.Bognár, G.Vincze, O.Benczenleitner (2005) Talent and talent development in sport: The issue of five sports. Kaloka., 3:113-123.

13. Géczi G., G.Vincze, M.Koltai, J.Bognár (2009) Elite young team sport players' coping, motivation and perceived climate measures. Physic. Cult. Sport Stud. Res., 229-242.

14. Grant H., C.S.Dweck (2003) Clarifying achievement goals and their impact. J. Pers. Soc. Psychol., 85,:541-553.

15. Hassandra M., M.Goudas, S.Chroni (2003) Examining the factors associated with intrinsic motivation in physiccal education: A qualitative approach. Psychol. Sport Exer., 4:211-223.
16. Jowett S. (2006) Interpersonal and Structural Features of Greek Coach-Athlete Dyads Performing in Individual Sports. J. Appl. Sport Psychol., 18:69-81.

17. Judge L.W. (2008) The complete track and field coaches' guide to conditioning for the throwing events. Monterey, CA: Coaches Choice Publishing.

18. Judge L.W., D.Bellar, G.McAtee, M. Judge (2010) Predictors of Personal Best Performance in the Hammer Throw for U.S. Collegiate Throwers. Int. J. Perf. Anal. Sport, 10(1):54-65.

19. Newton M., J.Duda, Yin Zenong (2000) Examination of the psychometric properties of the Perceived Motivational Climate in Sport Questionnaire-2 in a sample of female athletes. J. Sport. Sci., 18:275-290.

20. Pelletier L.G., M.Fortier, R.J.Vallerand, N.M. Briere, K.M. Tuson, M.R. Blais (1995) The Sport Motivation Scale (SMS-28). J. Sport Exer. Psychol.. Vol. 15:35-53.

21. Petherick C., D.Weigand, (2002) The relationship of dispositional goal orientations and perceived motivational climates on indices of motivation in male and female swimmers. Int. J. Sport Psychol., 33:218-237.

22. Révész L., J.Bognár, G.Trzaskoma, Bicsérdy, T.L.Kovács (2009) Validation of Perceived Motivational Climate in Sport Questionnaire-2 (PMCSQ-2) for Hungarian research [in Hungarian]. Kaloka., 2009.2-3. Budapest

23. Rojas-Ruiz F.J., M. Gutiérrez-Dávila (2009) The relation between angular displacement of the hammer in the double sport phase and its velocity in the hammer throw. J. Hum. Sport Exer, 4, 3:254-261.

24. Ryan R.M., E.L.Deci (2000) Self-determination theory and the facilitation of intrinsic motivation, social development, and well-being. Am. Psychol, 55:68-78.

25. Thomas K.T., J.Thomas (1999) What squirrels in the trees predict about expert athletes. Int. J. Sport Psychol., 30: 221-234.

26. Tsang Szabó, Soós, Bute, (2005) A Study of Cultural Differences in Motivational Orientations towards Sport Participation of Junior Secondary School Children in Four Cultures. J. Physic. Educ. Recreat., Vol. 11/1:44-50.

27. Viira R., L.Raudsepp (2000) Achievement goal orientations, beliefs about sport success and sport emotions as related to moderate to vigorous physical activity of adolescents. Psychol. Heal., 15:625-633.

28. Vlachopoulos S.P.; C.I.Karageorghis, P.C.Terry (2000) Motivation profiles in sport: a self-determination theory perspective. Res. Quart. of Exer. Sport, 71(4):387-97.

29. White S.A., J.L.Duda, M.R.Keller (1998) The relationship between goal orientation and perceived purposes of sport among youth sport participants. J. Sport Behav., 21, 4, 474-483.

Received 16.10.2012

Accepted 24.04.2013

(c) University of Physical Education, Warsaw, Poland 